\title{
ATTAINABILITY ANALYSIS IN THE PROBLEM OF STOCHASTIC EQUILIBRIA SYNTHESIS FOR NONLINEAR DISCRETE SYSTEMS
}

\author{
IRINA BASHKIRTSEVA, LEV RYASHKO
}

Institute of Mathematics and Computer Science

Ural Federal University, 51 Lenin Street, Ekaterinburg, Russia e-mail: lev.ryashko@usu.ru

\begin{abstract}
A nonlinear discrete-time control system forced by stochastic disturbances is considered. We study the problem of synthesis of the regulator which stabilizes an equilibrium of the deterministic system and provides required scattering of random states near this equilibrium for the corresponding stochastic system. Our approach is based on the stochastic sensitivity functions technique. The necessary and important part of the examined control problem is an analysis of attainability. For 2D systems, a detailed investigation of attainability domains is given. A parametrical description of the attainability domains for various types of control inputs in a stochastic Henon model is presented. Application of this technique for suppression of noise-induced chaos is demonstrated.
\end{abstract}

Keywords: stochastic sensitivity function, attainability, chaos suppression.

\section{Introduction}

An investigation of recently discovered unexpected phenomena in mechanics, chemical kinetics and biophysics is connected with the analysis of corresponding nonlinear dynamical models (Nayfeh and Balachandran, 2006; Fedotov et al., 2004; Alexandrov and Malygin, 2011). Numerous control nonlinear systems operate in zones of transition from order to chaos. After the pioneering work of Ott et al. (1990), controlling chaos has focused attention of many researchers. Various approaches to the solution of problems connected with both suppression and generation of chaos have been proposed (Chen and Yu, 2003; Fradkov and Pogromsky, 1998). For nonlinear dynamical systems with multiple coexisting attractors, even small stochastic disturbances can induce random transitions between the basins of attraction and generate such phenomena as stochastic resonance (McDonnell et al., 2008), noise-induced order (Gassmann, 1997; Matsumoto and Tsuda, 1983) or noise-induced chaos (Gao et al., 1999). Control theory for stochastic linear systems is well developed (Åström, 1970; Kučera, 1973). An analysis of attainability in the state space for these systems was presented by Digailova and Kurzhanskii (2004).

Control of nonlinear systems with regular and chaotic oscillations is a challenging and fundamental problem of modern nonlinear engineering (Sanjuan and Grebogi, 2010). Analysis of the stochastic stability and controllability of nonlinear dynamical systems seems to attract the attention of researchers (Ryashko, 1996; Karthikeyan and Balachandran, 2011; Zhirabok and Shumsky, 2012).

In this paper, we investigate the control problem for a nonlinear discrete-time stochastic system. The aim of control is the synthesis of a regulator guaranteeing the deterministic stability of the equilibrium and providing a required dispersion of random states of a stochastically forced system near this equilibrium. A detailed description of the stochastic attractor is given by the stationary probabilistic density function. Unfortunately, this function can be found analytically only for the simplest one-dimensional cases. Thus, asymptotics and approximations are widely used. For continuous-time systems, approximations based on stochastic sensitivity functions were proposed by Mil'shtein and Ryashko (1995) as well as Bashkirtseva and Ryashko (2000). The stochastic sensitivity functions technique was successfully applied to the analysis of stochastic 3D attractors (Ryagin and Ryashko, 2004; Bashkirtseva and Ryashko, 2009), backward stochastic bifurcations in the Roessler system (Bashkirtseva et al., 2010), and an analysis of the 
excitability for the FitzHugh-Nagumo model (Ryashko and Bashkirtseva, 2011a).

For discrete-time stochastic systems with small noise, an asymptotic analysis based on the stochastic sensitivity functions technique was worked out by Bashkirtseva et al. (2009).

For control systems, by the corresponding choice of the regulator one can change the stochastic sensitivity of randomly forced attractors. This approach was used by Bashkirtseva and Ryashko (2005) for the solution of the problem of controlling chaos in Brusselator.

A general theoretical method for the synthesis of a required sensitivity of discrete-time stochastic systems was proposed by Ryashko and Bashkirtseva (2011b). A practical realization of this method necessitates a constructive description of attainability domains. Our paper focuses on the investigation of attainability domains and their dependence on the geometry of control inputs.

In Section 2, we give a necessary mathematical background of the stochastic sensitivity function technique (Bashkirtseva et al., 2009) and its application for the control problem introduced by Ryashko and Bashkirtseva (2011b). For the two-dimensional case, a method of the geometrical description of the probability distribution for the stochastic equilibrium based on dispersion ellipses is presented. In Section 3, we give a detailed description of attainability domains for 2D systems. In Section 4, the stochastically forced Henon system is used for the demonstration of the main ideas and constructive methods of our theory. For three different types of control input, a geometrical description of attainability domains is given. We show constructively how these domains can be used for the stabilization of stochastic equilibria and suppression of noise-induced chaos.

\section{Stochastic sensitivity analysis and control}

Consider a nonlinear stochastic system

$$
x_{t+1}=f\left(x_{t}\right)+\varepsilon \sigma\left(x_{t}\right) \xi_{t}, \quad t=0,1, \ldots,
$$

where $x$ is an $n$-vector, $f(x)$ is a continuously differentiable $n$-vector function, $\sigma(x)$ is a continuous $n \times$ $m$-matrix function, $\xi_{t}$ is an $m$-dimensional uncorrelated random process with the parameters

$$
\mathrm{E} \xi_{t}=0, \quad \mathrm{E} \xi_{t} \xi_{t}^{\top}=\mathrm{I}, \quad \mathrm{E} \xi_{t} \xi_{k}^{\top}=0 \quad(t \neq k),
$$

where $I$ is the $m \times m$ identity matrix, $\varepsilon \geq 0$ is a scalar parameter of the noise intensity. It is supposed that the corresponding deterministic system (1) $(\varepsilon=0)$ has an exponentially stable equilibrium $x_{t} \equiv \bar{x}$

Let $x_{t}^{\varepsilon}$ be a solution of the system (1) with the initial condition $x_{0}^{\varepsilon}=\bar{x}+\varepsilon z_{0}$. The variable

$$
z_{t}=\lim _{\varepsilon \rightarrow 0} \frac{x_{t}^{\varepsilon}-\bar{x}}{\varepsilon}
$$

characterizes the sensitivity of the equilibrium $\bar{x}$ both to initial data disturbances and random disturbances of the system (1). For the sequence $z_{t}$, we have

$$
z_{t+1}=A z_{t}+G \xi_{t}, \quad A=\frac{\partial f}{\partial x}(\bar{x}), \quad G=\sigma(\bar{x}) .
$$

The dynamics of the second moments $Z_{t}=\mathrm{E} z_{t} z_{t}^{\top}$ for the solution $z_{t}$ of the system (2) are governed by the matrix equation

$$
Z_{t+1}=A Z_{t} A^{\top}+S, \quad S=G G^{\top} .
$$

The inequality $\rho(A)<1$, where $\rho(A)$ is the spectral radius of the matrix $A$, is a necessary and sufficient condition for the exponential stability of the equilibrium $\bar{x}$ (Elaydi, 1999). From the inequality $\rho(A)<1$ it follows that for any $Z_{0}$ we have $\lim _{t \rightarrow \infty} Z_{t}=W$, where the matrix $W$ is a unique solution of the Lyapunov equation

$$
W=A W A^{\top}+S
$$

For nonsingular noise $(\operatorname{rank}(S)=n)$, the matrix $W$ is positive definite.

Let the system (1) have a stochastic attractor defined by the solution $\bar{x}_{t}^{\varepsilon}$ with a stable stationary probabilistic distribution $p(x, \varepsilon)$. For small Gaussian noise, the stationary probability density function $p(x, \varepsilon)$ of the random states of the system (1) can be approximated by the normal distribution

$$
p(x, \varepsilon) \approx \frac{\exp \left(-\frac{1}{2 \varepsilon^{2}}(x-\bar{x})^{\top} W^{-1}(x-\bar{x})\right)}{\varepsilon \sqrt{(2 \pi)^{n} \operatorname{det}(W)}} .
$$

Using the matrix $W$, one can approximate the dispersion of random states $\bar{x}_{t}^{\varepsilon}$ around the deterministic equilibrium $\bar{x}$ as follows:

$$
\mathrm{E}\left(\bar{x}_{t}^{\varepsilon}-\bar{x}\right)\left(\bar{x}_{t}^{\varepsilon}-\bar{x}\right)^{\top} \approx \varepsilon^{2} W .
$$

Thus, the matrix $W$ is a simple quantitative characteristic of the response of the nonlinear system (1) to small random disturbances in a neighborhood of the equilibrium $\bar{x}$. This matrix $W$ plays the role of the stochastic sensitivity factor of the equilibrium $\bar{x}$.

Here, the theory of stochastic sensitivity of the equilibrium will be presented shortly. Detailed discussions and proofs can be found in the work of Bashkirtseva et al. (2009).

For the two-dimensional case, the stochastic sensitivity matrix $W$ has eigenvalues $\lambda_{1} \geq \lambda_{2} \geq 0$ and corresponding eigenvectors $w_{1}, w_{2}$. The eigenvalues $\lambda_{1}$ and $\lambda_{2}$ are convenient scalar characteristics of the stochastic sensitivity and determine dispersion values of random states of the system (1) in the directions $w_{1}$ and $w_{2}$. Consider projections $\eta_{i}=\left(\bar{x}_{t}^{\varepsilon}-\bar{x}, w_{i}\right)$ of the deviations of stationary distributed random states $\bar{x}_{t}^{\varepsilon}$ from 
the equilibrium $\bar{x}$ onto vectors $w_{i}$. For $\eta_{i}$ we have simple approximations $\mathrm{E} \eta_{i}^{2} \approx \varepsilon^{2} \lambda_{i}$. Thus, the values $\lambda_{1,2}$ along with eigenvectors $w_{i}$ characterize the form and size of the confidence ellipse

$$
\frac{\alpha_{1}^{2}}{\lambda_{1}}+\frac{\alpha_{2}^{2}}{\lambda_{2}}=2 \varepsilon^{2} \beta^{2}, \quad \beta^{2}=-\ln (1-p) .
$$

Here $p$ is the value of the fiducial probability, $\alpha_{i}=(x-$ $\left.\bar{x}, w_{i}\right)$.

Consider now a controlled stochastic system,

$$
x_{t+1}=f\left(x_{t}, u_{t}\right)+\varepsilon \sigma\left(x_{t}, u_{t}\right) \xi_{t},
$$

where $x$ is an $n$-vector, $f(x, u)$ is a continuously differentiable $n$-vector function, $u$ is an $l$-vector of control parameters, $\sigma(x, u)$ is a sufficiently smooth $n \times m$-matrix function characterizing the dependence of disturbances on state and control, $\xi_{t}$ is an uncorrelated random process with parameters $\mathrm{E} \xi_{t}=0, \mathrm{E} \xi_{t} \xi_{t}^{\top}=\mathrm{I}, \mathrm{E} \xi_{t} \xi_{k}=0(t \neq$ $k), \varepsilon$ is a scalar parameter of noise intensity. It is supposed that for $u=0, \varepsilon=0$ the system (8) has an equilibrium $x_{t} \equiv \bar{x}$. A stability of $\bar{x}$ is not assumed.

Consider a class $\mathcal{U}$ of admissible feedbacks $u=$ $u(x)$ satisfying the following conditions:

(a) $u(x)$ is continuously differentiable and $u(\bar{x})=0$;

(b) for the closed-loop deterministic system

$$
x_{t+1}=f\left(x_{t}, u\left(x_{t}\right)\right)
$$

the equilibrium $\bar{x}$ is exponentially stable.

Consider a set of matrices

$$
\mathbb{K}=\left\{K \in \mathbb{R}^{l \times n}, \quad \rho(A+B K)<1\right\},
$$

where

$$
A=\frac{\partial f}{\partial x}(\bar{x}, 0), \quad B=\frac{\partial f}{\partial u}(\bar{x}, 0), \quad K=\frac{\partial u}{\partial x}(\bar{x}) .
$$

Suppose that the pair $(A, B)$ is stabilizable (Wohnam, 1979). This means that neither set $\mathbb{K}$ nor class $\mathcal{U}$ is empty.

For any $K \in \mathbb{K}$, one can find the stochastic sensitivity matrix $W$ for the equilibrium $\bar{x}$. This matrix is a unique solution of the equation

$$
\begin{aligned}
W & =(A+B K) W(A+B K)^{\top}+S, \\
S & =G G^{\top}, \quad G=\sigma(\bar{x}, 0) .
\end{aligned}
$$

Note that the matrix $W$ is completely determined by the linear approximation of the function $u(x)$ and independent of higher-order terms. This allows us to restrict the study without loss of generality to more simple regulators in the form of linear feedback,

$$
u(x)=K(x-\bar{x}) .
$$

Consider a set of admissible stochastic sensitivity matrices,

$$
\mathbb{M}=\left\{M \in \mathbb{R}^{n \times n} \mid M \succ 0\right\},
$$

where $M \succ 0$ means that the matrix $M$ is symmetric and positive definite.

Problem 1. (Stochastic sensitivity synthesis) For the assigned matrix $W \in \mathbb{M}$, it is necessary to find a matrix $K \in \mathbb{K}$ guaranteeing the equality $W_{K}=W$, where $W_{K}$ is a solution of Eqn. (9).

For some matrices $W \in \mathbb{M}$, this problem is unsolvable. Therefore, we use a notion of attainability introduced by Ryashko and Bashkirtseva (2011b).

Definition 1. An element $W \in \mathbb{M}$ is said to be attainable for the system (8) under the feedback (10) if the equality $W_{K}=W$ is true for some $K \in \mathbb{K}$.

Definition 2. A set of all attainable elements

$$
\mathbb{W}=\left\{W \in \mathbb{M} \mid \exists K \in \mathbb{K}, \quad W_{K}=W\right\}
$$

is called the attainability set for the system (8) and (10).

Attainability sets in the state space on a finite time interval of control were studied by Digailova and Kurzhanskii (2004) for linear stochastic systems. Our paper deals with the notion of attainability corresponding to the problem of control for stochastic attractors.

Let us describe an attainability set. A relation $Q \succeq$ $P$ means that the matrix $Q-P$ is non-negative definite. From Eqn. (9) it follows that $W_{K} \succeq S$ for any $A, B$ and $K$. This means that $\mathbb{W} \subseteq \mathbb{M}_{S}$, where $\mathbb{M}_{S}=\{V \in$ $\mathbb{M} \mid V \succeq S\}$. A solution to the problem of the synthesis of the stochastic sensitivity matrix is given by the following theorem (Ryashko and Bashkirtseva, 2011b).

Theorem 1. Let the noise in the system (8) be non$\operatorname{singular}(S \succ 0)$.

(a) If the matrix $B$ is quadratic and non-singular $(\operatorname{rank}(B)=n=l)$ then $\mathbb{W}=\mathbb{M}_{S}$ and for any matrix $W \in \mathbb{M}_{S}$, Eqn. (9) has a solution,

$$
K=B^{-1}\left[(W-S)^{\frac{1}{2}} U^{\top} W^{-\frac{1}{2}}-A\right] \in \mathbb{K},
$$

where $U$ is an arbitrary orthogonal $n \times n$-matrix.

(b) If $\operatorname{rank}(B)<n$, then the element $W \in \mathbb{M}_{S}$ is attainable if and only if the matrix $W$ is a solution of the equation

$$
P A W A^{\top} P=P(W-S) P,
$$

where $P=I-B B^{+}$is a projection matrix, '+' means a pseudoinversion (Albert, 1972). Under these conditions, 
for any matrix $W \in \mathbb{M}_{S}$ satisfying (12), Eqn. (9) has a solution,

$$
K=B^{+}\left[(W-S)^{\frac{1}{2}} U^{\top} W^{-\frac{1}{2}}-A\right] \in \mathbb{K} .
$$

Here $U$ is an orthogonal $n \times n$-matrix satisfying the condition

$$
P A W^{\frac{1}{2}}=P(W-S)^{\frac{1}{2}} U^{\top} .
$$

This theorem can be used for the construction of attainability sets. Indeed, these sets are described parametrically using a general solution of the matrix equation (12). In this paper, we focus on the application of this approach to the two-dimensional case and give a detailed description of attainability sets for various control inputs.

Remark 1. Using the presented theory, one can check the attainability of the assigned stochastic sensitivity matrix $W$. For the attainable matrices $W$, the formulas (11) and 13) yield an explicit value for the matrix $K$ of the feedback regulator (10) synthesizing $W$. Note that the stochastic sensitivity matrix $W$ is an asymptotic characteristic of the closed-loop nonlinear system (8), (10) for infinitesimal noise. For small noise, random states of a nonlinear system are localized in a small vicinity of the equilibrium. Due to this localization, parameters of the linear approximation system define $W$ quite adequately. For a small noise intensity $\varepsilon$, a dispersion of stationary distributed random states of the system (8), (10) around the equilibrium is approximated by (6). In particular, small values of the assigned $W$ yield small dispersion.

\section{Attainability analysis for a 2D system}

For a 2D system $(n=2)$ due to the symmetry of matrices $W$ and $S$, we have

$$
\begin{gathered}
A=\left[\begin{array}{ll}
a_{11} & a_{12} \\
a_{21} & a_{22}
\end{array}\right], \quad W=\left[\begin{array}{ll}
w_{11} & w_{12} \\
w_{12} & w_{22}
\end{array}\right], \\
S=\left[\begin{array}{ll}
s_{11} & s_{12} \\
s_{12} & s_{22}
\end{array}\right],
\end{gathered}
$$

where

$$
\begin{aligned}
a_{11} & =\frac{\partial f_{1}}{\partial x_{1}}(\bar{x}), & a_{12} & =\frac{\partial f_{1}}{\partial x_{2}}(\bar{x}), \\
a_{21} & =\frac{\partial f_{2}}{\partial x_{1}}(\bar{x}), & a_{22} & =\frac{\partial f_{2}}{\partial x_{2}}(\bar{x}) .
\end{aligned}
$$

First, consider the system (8), (10) with the non-degenerate $2 \times 2$-matrix $B: \operatorname{rank}(B)=2$. In this case, due to Theorem 1, the set of attainable matrices $\mathbb{W}$ coincides with the set $\mathbb{M}_{S}$. Elements of the attainable matrix $W$ satisfy the following system of three inequalities:

$$
\begin{aligned}
& w_{11} \geq s_{11}, \quad w_{22} \geq s_{22}, \\
& \left(w_{11}-s_{11}\right)\left(w_{22}-s_{22}\right) \geq\left(w_{12}-s_{12}\right)^{2} .
\end{aligned}
$$

For $w_{12}=s_{12}$, attainable diagonal elements of the matrix $W$ are bounded by $w_{11} \geq s_{11}$, and $w_{22} \geq s_{22}$.

For $w_{12} \neq s_{12}$, the attainability set can be described as

$$
w_{11}>s_{11}, \quad w_{22} \geq s_{22}+\frac{\left(w_{12}-s_{12}\right)^{2}}{w_{11}-s_{11}} .
$$

Geometrically, this means that the attainability domain is bounded from below by the corresponding hyperbola. Note that here the non-diagonal element $w_{12}$ of the attainable matrix $W$ can be chosen arbitrarily, but attainable diagonal elements $w_{11}$ and $w_{22}$ have to satisfy the inequalities written above. These inequalities give much freedom in choosing the elements of $W$. In the problem of suppression of large-amplitude stochastic oscillations, it is reasonable to use a regulator providing minimal values of stochastic sensitivity. In the case considered, these values are $w_{11}=s_{11}, \quad w_{12}=$ $s_{12}, w_{22}=s_{22}$.

Second, consider the system (8), (10) with the $2 \times$ 1-matrix $B: \operatorname{rank}(B)=1$. Without loss of generality, we can set

$$
B=\left[\begin{array}{l}
0 \\
1
\end{array}\right] \text {. }
$$

In this case, an attainability set decreases: from (13) an additional restriction follows,

$$
\left(a_{11}^{2}-1\right) w_{11}+2 a_{11} a_{12} w_{12}+a_{12}^{2} w_{22}+s_{11}=0 .
$$

Here a pair $(A, B)$ is controllable (Wonham, 1979) if and only if

$$
\operatorname{rank}[B, A B]=\operatorname{rank}\left[\begin{array}{cc}
0 & a_{12} \\
1 & a_{22}
\end{array}\right]=2
$$

This holds if $a_{12} \neq 0$.

Consider two subcases.

Case 1. $a_{12} \neq 0, a_{11} \neq 0$. From (16) it follows that

$$
w_{12}=\frac{\left(1-a_{11}^{2}\right) w_{11}-a_{12}^{2} w_{22}-s_{11}}{2 a_{11} a_{12}} .
$$

Substituting $w_{12}$ into 15 we have

$$
\begin{aligned}
& w_{11} \geq s_{11}, \quad w_{22} \geq s_{22}, \\
& w_{22}^{2}+p\left(w_{11}\right) w_{22}+q\left(w_{11}\right) \leq 0,
\end{aligned}
$$

where

$$
\begin{aligned}
& p\left(w_{11}\right) \\
& =\frac{-2\left[\left(1+a_{11}^{2}\right) w_{11}-\left(1+2 a_{11}^{2}\right) s_{11}-2 a_{11} a_{12} s_{12}\right]}{a_{12}^{2}}, \\
& q\left(w_{11}\right) \\
& =\frac{s_{11}^{2}+4 a_{11}^{2} a_{12}^{2}\left(s_{12}^{2}-s_{11} s_{22}\right)+4 a_{11} a_{12} s_{11} s_{12}}{a_{12}^{4}} .
\end{aligned}
$$


The system (18) defines the attainability domain for the diagonal elements $w_{11}$ and $w_{22}$ of matrix $W$. For any attainable pair $w_{11}, w_{22}$, the non-diagonal element $w_{12}$ can be found uniquely from (17).

Case 2. $a_{12} \neq 0, a_{11}=0$. From (16) it follows that

$$
w_{22}=\frac{w_{11}-s_{11}}{a_{12}^{2}} .
$$

Substituting $w_{22}$ into (15) we have

$$
\begin{aligned}
w_{11} & \geq s_{11}, \\
w_{11}^{2}- & \left(2 s_{11}+a_{12}^{2} s_{22}\right) w_{11} \\
& +s_{11}\left(s_{11}+a_{12}^{2} s_{22}\right) \geq a_{12}^{2}\left(w_{12}-s_{12}\right)^{2} .
\end{aligned}
$$

Here, for any $w_{12}$, one can find attainable $w_{11}$ from (20) and $w_{22}$ from (19).

Consider a case $a_{12}=0$ when the pair $(A, B)$ is not controllable. The stabilizability condition implies $a_{11}^{2}<$ 1. From (15) and (16) it follows that

$$
w_{11}=\frac{s_{11}}{1-a_{11}^{2}}, \quad w_{22} \geq s_{22}+\frac{\left(w_{12}-s_{12}\right)^{2}\left(1-a_{11}^{2}\right)}{s_{11} a_{11}^{2}} .
$$

Among attainable $w_{22}$ the minimal value is $w_{22}=s_{22}$ for $w_{12}=s_{12}$.

Constructive possibilities of this attainability analysis are demonstrated for the Henon system in the next section.

\section{Control of a stochastic Henon system}

Consider the stochastically forced Henon system (Henon, 1976)

$$
\begin{aligned}
& x_{t+1}=1-\mu x_{t}^{2}-0.5 y_{t}+\varepsilon \sigma_{1} \xi_{t}, \\
& y_{t+1}=x_{t}+\varepsilon \sigma_{2} \nu_{t}, \quad 1 \leq \mu \leq 2.4,
\end{aligned}
$$

where $\xi_{t}, \nu_{t}$ are sequences of independent Gaussian random disturbances with parameters $E \xi_{t}=E \nu_{t}=0$, $E \xi_{t}^{2}=1, E \nu_{t}^{2}=1, E \xi_{t} \nu_{t}=0$, and $\varepsilon$ is a scalar parameter of the noise intensity.

The corresponding deterministic model 21) $(\varepsilon=0)$ has the equilibrium $(\bar{x}, \bar{y})$, where

$$
\bar{x}=\bar{y}=\frac{\sqrt{9+16 \mu}-3}{4 \mu} .
$$

The stability of the equilibrium is defined by the criterion $\rho(A)<1$. For the Henon system,

$$
A=\left[\begin{array}{cc}
a & -0.5 \\
1 & 0
\end{array}\right]
$$

with $a=-2 \mu \bar{x}=(3-\sqrt{9+16 \mu}) / 2$. The eigenvalues of the matrix $A$ are solutions of the following equation:

$$
\lambda^{2}+\frac{1}{2}(\sqrt{9+16 \mu}-3) \lambda+\frac{1}{2}=0 .
$$

Here the criterion of the stability of the equilibria $(\bar{x}, \bar{y})$ is given by

$$
\mu<\mu_{*}=\frac{27}{16}=1.6875 .
$$

For $\mu \in[1,1.6875)$ this equilibrium is exponentially stable. When the parameter $\mu$ passes the bifurcation value $\mu_{*}=1.6875$, this equilibrium loses stability. On the interval $(1.6875,2.5]$, the Henon system demonstrates both periodic and chaotic oscillations.

For a small noise intensity $(\varepsilon>0)$, the stochastically forced trajectories of the system (21) form stochastic attractors around the corresponding deterministic ones.

Consider a zone $\mu \in[1,1.6875)$ of the stochastic equilibria. A plot of the dispersion $D(\mu)=E\left[\left(\bar{x}_{t}^{\varepsilon}-\right.\right.$ $\left.\bar{x})^{2}+\left(\bar{y}_{t}^{\varepsilon}-\bar{y}\right)^{2}\right]$ of the stationary distributed random states $\left(\bar{x}_{t}^{\varepsilon}, \bar{y}_{t}^{\varepsilon}\right)$ calculated by direct numerical simulation in this zone is presented in Fig. 1 by circles for $\varepsilon=0.005, \sigma_{1}=$ $\sigma_{2}=1$. As one can see, the function $D(\mu)$ monotonically grows and changes by more than two orders.

In Figs. 2 and 7(a)-(b) the random states (grey) of the stochastic Henon system for different values of the parameter $\mu$ are plotted. Stochastically forced equilibria for $\mu=1.1$ and $\mu=1.6$ are presented in Fig. 2. In Fig. 7(a), one can see a stochastically forced 2-cycle for $\mu=2$. For $\mu=2.4$, the deterministic Henon system has a stable 4-cycle. Under the random disturbances, this 4-cycle transforms to the stochastic attractor which looks chaotic (see Fig. 7(b)).

Our probabilistic analysis of the stochastic equilibria is based on the stochastic sensitivity matrix technique (see Section 2). For the Henon system (21), due to (4), elements of the stochastic sensitivity matrix

$$
W=\left[\begin{array}{ll}
w_{11} & w_{12} \\
w_{21} & w_{22}
\end{array}\right]
$$

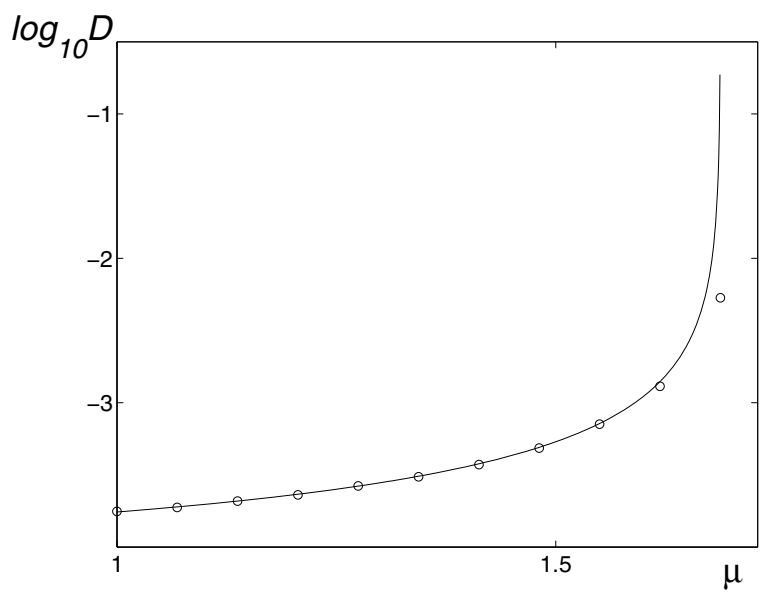

Fig. 1. Dispersion of random states for the Henon model 21 for $\varepsilon=0.005, s_{11}=s_{22}=1$. 
satisfy the following system:

$$
\begin{aligned}
& w_{11}=a^{2} w_{11}-a w_{12}+0.25 w_{22}+s_{11}, \\
& w_{12}=w_{21}=a w_{11}-0.5 w_{12}, \\
& w_{22}=w_{11}+s_{22},
\end{aligned}
$$

where $s_{11}=\sigma_{1}^{2}, s_{22}=\sigma_{2}^{2}, s_{12}=0$. An explicit solution of this system is the following:

$$
\begin{gathered}
w_{11}=\frac{12 s_{11}+3 s_{22}}{9-4 a^{2}}, \quad w_{22}=\frac{12 s_{11}+3 s_{22}}{9-4 a^{2}}+s_{22}, \\
w_{12}=\frac{2 a\left(4 s_{11}+s_{22}\right)}{9-4 a^{2}} .
\end{gathered}
$$

Using the elements of this stochastic sensitivity matrix $W$, we can approximate the dispersion function $D(\mu) \approx$ $D_{s}(\mu)=\varepsilon^{2}\left(w_{11}+w_{22}\right)$. For $\varepsilon=0.005, s_{11}=s_{22}=1$, in Fig. 1 the curve $D_{s}(\mu)$ is plotted with a solid line. As we can see, the function $D_{s}(\mu)$ yields an adequate approximation of the dispersion $D(\mu)$.

The stochastic sensitivity matrix technique allows us not only to find a dispersion, but to analyze a spatial arrangement of random states as well. Using the eigenvalues and eigenvectors of the stochastic sensitivity matrix $W$, one can present details of the probabilistic distribution of the stochastic equilibria via the confidence ellipses (7). In Fig. 2, for $\varepsilon=0.005, s_{11}=s_{22}=1$, fiducial probability $p=0.95$ and two values $\mu=1.1$ and $\mu=1.6$, the confidence ellipses (7) and random states for the Henon model 21) are plotted. As we can see, the confidence ellipses descriptively reflect the details of the configurational arrangement for random states.

The Henon map is a canonical model for systems that exhibit various nonlinear phenomena. This model

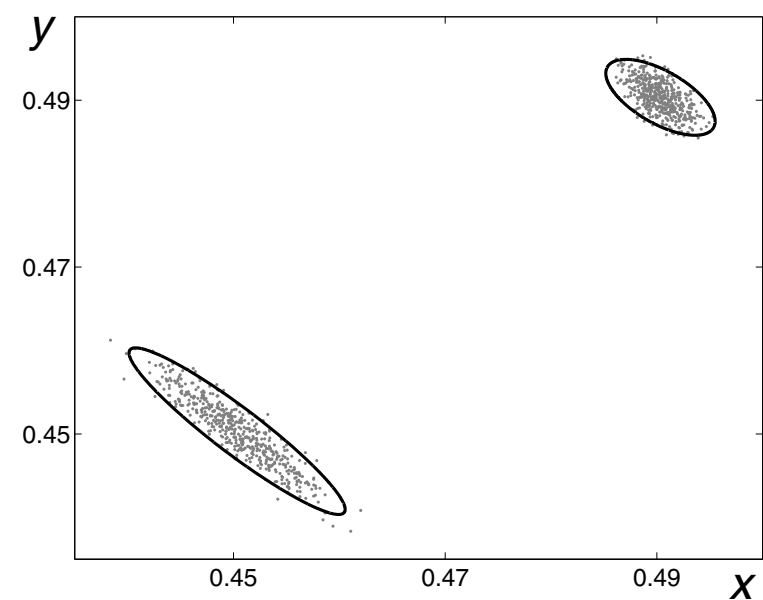

Fig. 2. Random states and confidence ellipses for the uncontrolled stochastic Henon model (21) with $\varepsilon=$ $0.001, s_{11}=s_{22}=1$, fiducial probability $p=0.95$ for $\mu=1.1$ (right) and $\mu=1.6$ (left). is actively used by researchers to study different bifurcations, noise-induced phenomena and control methods. Transformation of complex multistability to controlled monostability for a stochastically forced Henon model was investigated by Goswami and Basu (2002). For the Henon model, it was shown that stochastic control of attractor preference can be used to modify the global structure of coexisting attractors and their basins of attraction (Martínez-Zéregaa and Pisarchik, 2002).

Now, we are going to apply the stochastic sensitivity functions technique to constructive solution of the control problem for stochastic attractors of the Henon system.

Consider three variants of control inputs. First (Control I), the system (21) is governed by two independent control inputs $u_{1}, u_{2}$. Second (Control II), the control $u_{1}$ acts on the first equation of (21) only. Third (Control III), the control $u_{2}$ acts on the second equation only. These three cases allow clarifying the main statements of our theory from Sections 2 and 3 and show constructive possibilities of its applications.

4.1. Control I. Consider the stochastically forced Henon system

$$
\begin{aligned}
& x_{t+1}=1-\mu x_{t}^{2}-0.5 y_{t}+u_{1, t}+\varepsilon \sigma_{1} \xi_{t}, \\
& y_{t+1}=x_{t}+u_{2, t}+\varepsilon \sigma_{2} \nu_{t}
\end{aligned}
$$

with control inputs $u_{1, t}$ and $u_{2, t}$. The aim of the control is to provide stability of equilibria on the whole interval $1 \leq$ $\mu \leq 2.4$ and synthesize the required stochastic sensitivity matrix $W$.

We use a feedback regulator in the following form:

$$
\begin{aligned}
& u_{1}=k_{11}(x-\bar{x})+k_{12}(y-\bar{y}), \\
& u_{2}=k_{21}(x-\bar{x})+k_{22}(y-\bar{y}) .
\end{aligned}
$$

Due to Theorem 1, for the system (22), (23), the elements of attainable matrices $W$ are restricted by the inequalities (15),

$$
\begin{gathered}
w_{11} \geq s_{11}, \quad w_{22} \geq s_{22}, \\
\left(w_{11}-s_{11}\right)\left(w_{22}-s_{22}\right) \geq w_{12}^{2} .
\end{gathered}
$$

The attainability sets for cases $w_{12}=0$ and $w_{12} \neq 0$ are demonstrated by crosshatched regions in Figs. 3(a) and 3 (b) respectively.

Set $w_{12}=0$. This choice leads to the lack of correlation of random states $\bar{x}_{t}^{\varepsilon}$ and $\bar{y}_{t}^{\varepsilon}$. Then the elements $w_{11}$ and $w_{22}$ of attainable matrices $W$ are restricted by the inequalities

$$
w_{11} \geq s_{11}, \quad w_{22} \geq s_{22} .
$$

As follows from (11), the coefficients of the feedback regulator (23) guaranteeing the required values $w_{11} \geq$ 


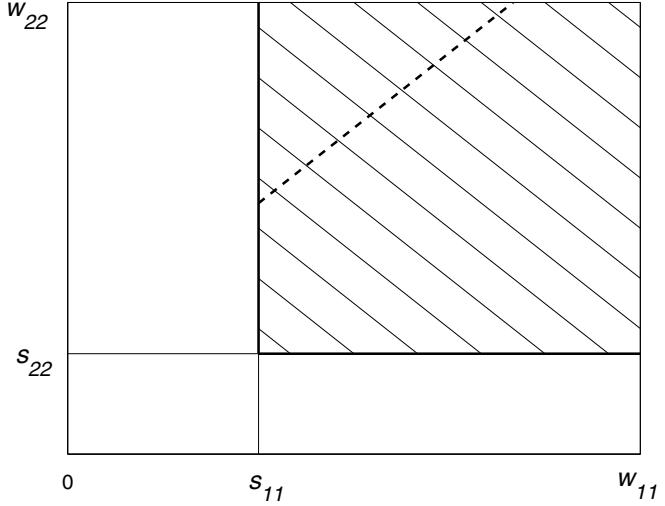

(a)

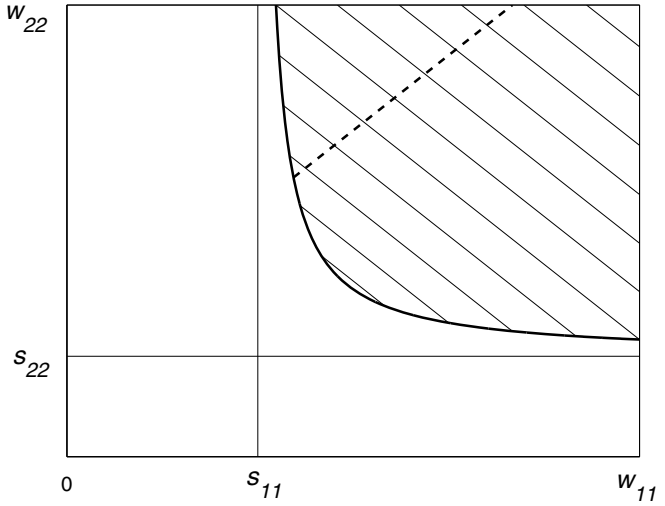

(b)

Fig. 3. Attainability zones: crosshatched region (Henon model (22) $)$ and dashed line (Henon model (24) $)$ for $w_{12}=0$ (a), $w_{12} \neq 0$ (b).

$s_{11}, w_{12}=0, w_{22} \geq s_{22}$ of the stochastic sensitivity matrix can be calculated by the formulas

$$
\begin{gathered}
k_{11}=\sqrt{1-\frac{s_{11}}{w_{11}}}-a, \quad k_{12}=0.5, \\
k_{21}=-1, \quad k_{22}=\sqrt{1-\frac{s_{22}}{w_{22}}} .
\end{gathered}
$$

Here $U=I$.

Possibilities of this regulator to synthesize stochastic attractors with a different spatial form are demonstrated in Fig. 4.

We consider four variants of the required stochastic sensitivity matrices:

$$
\begin{gathered}
W_{1}=\left[\begin{array}{ll}
1 & 0 \\
0 & 1
\end{array}\right], W_{2}=\left[\begin{array}{cc}
10 & 0 \\
0 & 1
\end{array}\right], \\
W_{3}=\left[\begin{array}{cc}
1 & 0 \\
0 & 10
\end{array}\right], W_{4}=\left[\begin{array}{cc}
10 & 9 \\
9 & 10
\end{array}\right] .
\end{gathered}
$$

Random states of the Henon system (22) for $\mu=1.6, \varepsilon=$ $0.001, \sigma_{1}=\sigma_{2}=1$ with controls synthesizing these required sensitivity matrices are presented in Fig. 4: (a) for $W_{1}$, (b) for $W_{2}$, (c) for $W_{3}$, (d) for $W_{4}$. Note that the matrix $W_{1}$ is a minimal element in the attainability set. For the matrix $W_{1}$, a dispersion of random states is minimal, too. As a result of the corresponding control, random states of the system (22) are uniformly concentrated near equilibria (see Fig. 4(a)) while random states of the uncontrolled system (21) are widely dispersed (see Fig. 2, left). In this case, our control compresses the confidence ellipse of uncontrolled systems and transforms it to a circle. Varying the required stochastic sensitivity matrices, we can synthesize dispersion ellipses of different configurations (see Fig. 4(b)-(d)). Because of the attainability conditions, all these ellipses contain the circle from Fig. 4(a).
Note that, for $W \neq S$, a matrix $K$ of feedback coefficients in (11) depends on the arbitrary orthogonal matrix $U$. Let $W=2 S$. For the system (21) with $\sigma_{1}=\sigma_{2}=1$, this means that

$$
\begin{gathered}
W=\left[\begin{array}{ll}
2 & 0 \\
0 & 2
\end{array}\right], \quad W^{\frac{1}{2}}=\left[\begin{array}{cc}
\sqrt{2} & 0 \\
0 & \sqrt{2}
\end{array}\right], \\
(W-S)^{\frac{1}{2}}=\left[\begin{array}{ll}
1 & 0 \\
0 & 1
\end{array}\right], \quad U=\left[\begin{array}{cc}
\cos \varphi & -\sin \varphi \\
\sin \varphi & \cos \varphi
\end{array}\right] .
\end{gathered}
$$

Here $U=U(\varphi)$ is a one-parametrical family of orthogonal $2 \times 2$-matrices.

As follows from (11), the feedback matrices $K$ synthesizing the stochastic sensitivity matrix $W$ form a one-parametrical family, too:

$$
K(\varphi)=\left[\begin{array}{cc}
\frac{\sqrt{2}}{2} \cos \varphi+2 \mu \bar{x} & \frac{\sqrt{2}}{2} \sin \varphi+\frac{1}{2} \\
-\frac{\sqrt{2}}{2} \sin \varphi-1 & \frac{\sqrt{2}}{2} \cos \varphi
\end{array}\right] .
$$

The control cost function $J=E u^{2}$ is

$$
\begin{aligned}
J(\varphi) & =\varepsilon^{2} \operatorname{tr}\left(K^{\top} K W\right) \\
& =\varepsilon^{2}\left[4 \sqrt{2} \mu \bar{x} \cos \varphi+3 \sqrt{2} \sin \varphi+8 \mu^{2} \bar{x}^{2}+\frac{9}{2}\right] .
\end{aligned}
$$

The function $J(\varphi)$ has a minimal value,

$$
J_{0}=\varepsilon^{2}\left(4.5+8 \mu^{2} \bar{x}^{2}-\sqrt{18+32 \mu^{2} \bar{x}^{2}}\right),
$$

for

$$
\varphi_{0}=\operatorname{atan}\left(\frac{3}{4 \mu \bar{x}}\right)
$$

4.2. Control II. Consider now the stochastically forced Henon system

$$
\begin{aligned}
& x_{t+1}=1-\mu x_{t}^{2}-0.5 y_{t}+u_{1, t}+\varepsilon \sigma_{1} \xi_{t}, \\
& y_{t+1}=x_{t}+\varepsilon \sigma_{2} \nu_{t},
\end{aligned}
$$




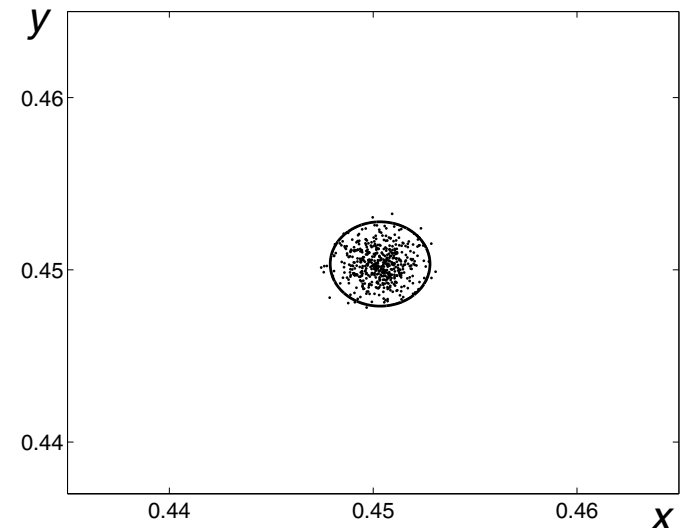

(a)

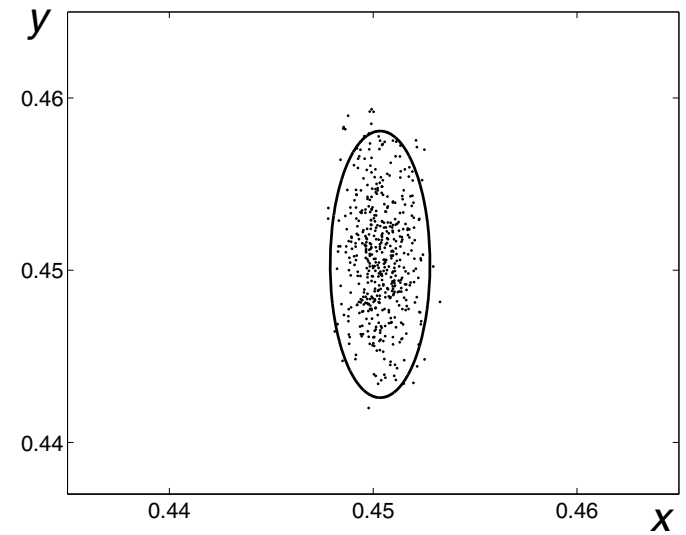

(c)

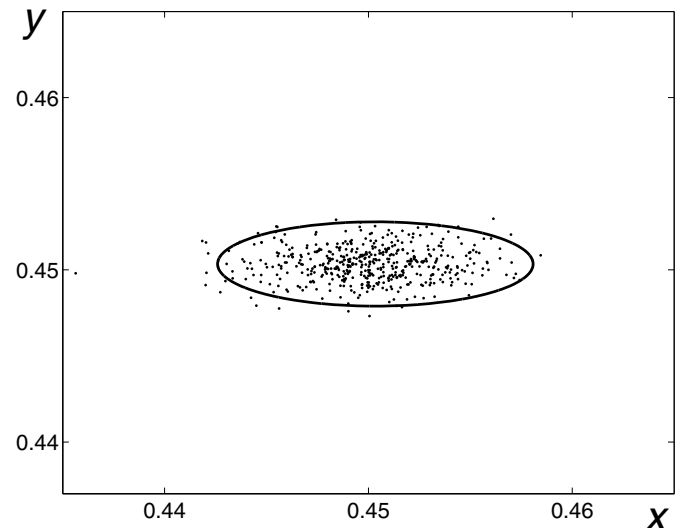

(b)

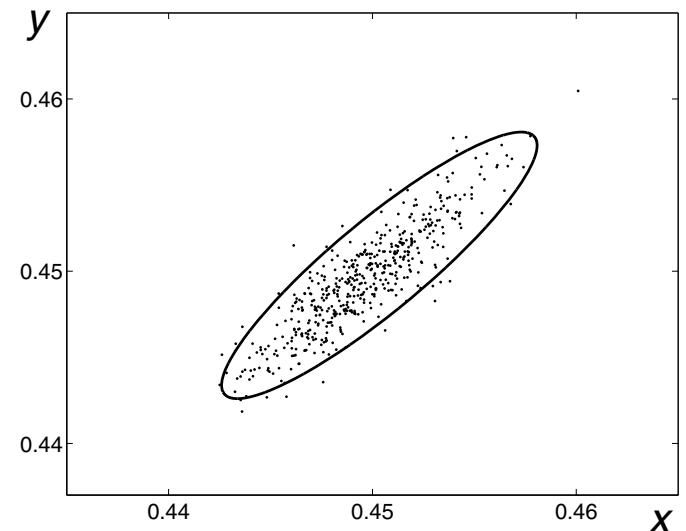

(d)

Fig. 4. Random states and confidence ellipses for the stochastic Henon model (22) with $\mu=1.6, \varepsilon=0.001, s_{11}=s_{22}=1$, fiducial probability $p=0.95$ and control inputs synthesizing different stochastic sensitivity matrices: $w_{11}=1, w_{22}=1, w_{12}=0$ (a), $w_{11}=10, w_{22}=1, w_{12}=0$ (b), $w_{11}=1, w_{22}=10, w_{12}=0$ (c), $w_{11}=10, w_{22}=10, w_{12}=9$ (d).

with a single control input $u_{1, t}$.

The aim of the control is to provide a stability of equilibria on the whole interval $1 \leq \mu \leq 2.4$ and synthesize the required stochastic sensitivity matrix $W$.

In this case,

$B=\left[\begin{array}{l}1 \\ 0\end{array}\right], \quad \operatorname{rank}[B, A B]=\operatorname{rank}\left[\begin{array}{cc}1 & -2 \mu \bar{x} \\ 0 & 1\end{array}\right]=2$,

so the pair $(A, B)$ is controllable.

We use a feedback regulator in the following form:

$$
u_{1}=k_{11}(x-\bar{x})+k_{12}(y-\bar{y}) .
$$

For the system 24, 25),

$$
\begin{gathered}
B^{+}=\left[\begin{array}{ll}
1 & 0
\end{array}\right], \quad B B^{+}=\left[\begin{array}{ll}
1 & 0 \\
0 & 0
\end{array}\right], \\
P=I-B B^{+}=\left[\begin{array}{ll}
0 & 0 \\
0 & 1
\end{array}\right] .
\end{gathered}
$$

Since $\operatorname{rank}(B)<2$, for attainability analysis and design of the feedback control we use Part (b) of Theorem 1. From the attainability condition (12) it follows that the elements of the assigned stochastic sensitivity matrix $W$ satisfy the system

$$
\begin{aligned}
& w_{11} \geq s_{11}, \quad\left(w_{11}-s_{11}\right)\left(w_{22}-s_{22}\right) \geq w_{12}^{2}, \\
& w_{22}=w_{11}+s_{22} .
\end{aligned}
$$

The attainability sets of the system (24), 25) for cases $w_{12}=0$ and $w_{12} \neq 0$ are plotted with dashed lines in Figs. 3(a) and (b), respectively. As we can see, the attainability set for the system (24), 25) with a single control input under the restriction 26 is essentially smaller than the attainability set for the system 22, (23) with two control inputs. Note that in these two cases attainability domains do not depend on the parameter $\mu$ of the deterministic Henon system. Set $w_{12}=0$. Then the elements $w_{11}$ and $w_{22}$ of attainable matrices $W$ are restricted by the relations

$$
w_{11} \geq s_{11}, \quad w_{22}=w_{11}+s_{22} .
$$




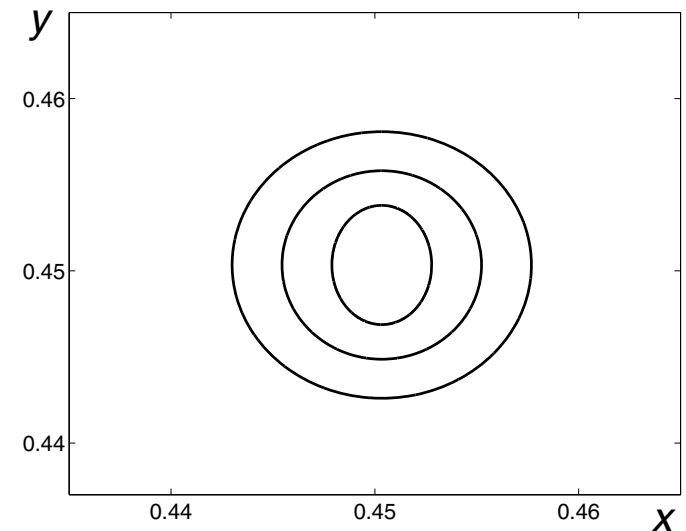

(a)

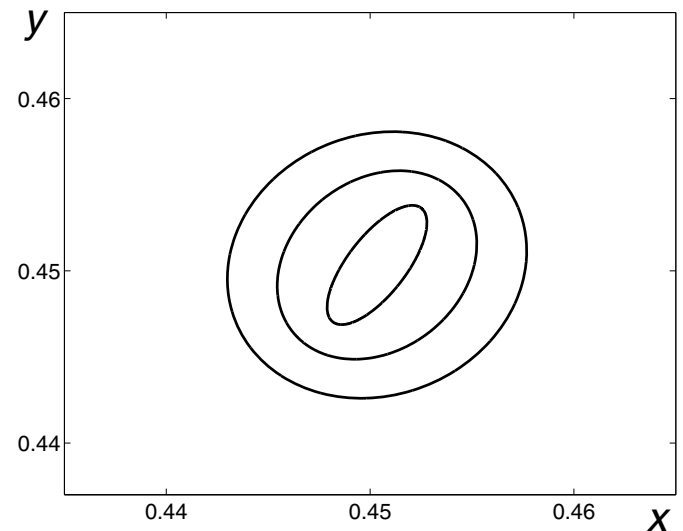

(b)

Fig. 5. Confidence ellipses for the stochastic Henon model (24) with $\mu=1.6, \varepsilon=0.001, s_{11}=s_{22}=1$, fiducial probability $p=$ 0.95 and control inputs synthesizing different stochastic sensitivity matrices $w_{11}=2, w_{22}=3$ (small), $w_{11}=4, w_{22}=5$ (middle), $w_{11}=9, w_{22}=10$ (large) for $w_{12}=0$ (a), $w_{12}=1$ (b).

Here

$$
\begin{gathered}
W^{\frac{1}{2}}=\left[\begin{array}{cc}
\sqrt{w_{11}} & 0 \\
0 & \sqrt{w_{11}+s_{22}}
\end{array}\right] \\
(W-S)^{\frac{1}{2}}=\left[\begin{array}{cc}
\sqrt{w_{11}-s_{11}} & 0 \\
0 & \sqrt{w_{11}}
\end{array}\right] .
\end{gathered}
$$

Now we have to find an orthogonal $2 \times 2$-matrix $U$ satisfying the condition (14). Let

$$
U=\left[\begin{array}{cc}
\cos \varphi & -\sin \varphi \\
\sin \varphi & \cos \varphi
\end{array}\right]
$$

Then

$$
\begin{gathered}
P A W^{\frac{1}{2}}=\left[\begin{array}{cc}
0 & 0 \\
\sqrt{w_{11}} & 0
\end{array}\right], \\
P(W-S)^{\frac{1}{2}} U^{\top}=\left[\begin{array}{cc}
0 & 0 \\
\sqrt{w_{11}} \sin \varphi & \sqrt{w_{11}} \cos \varphi
\end{array}\right] .
\end{gathered}
$$

As follows from (14), $\sin \varphi=1, \cos \varphi=0$, and the orthogonal matrix $U$ can be uniquely found:

$$
U=\left[\begin{array}{cc}
0 & -1 \\
1 & 0
\end{array}\right]
$$

Using this matrix in the formula 13, we get the coefficients

$$
k_{11}=2 \mu \bar{x}, \quad k_{12}=0.5-\sqrt{\frac{w_{11}-s_{11}}{w_{11}+s_{22}}}
$$

of the regulator 25 guaranteeing the required values of the stochastic sensitivity matrix for the system 24.

An analogous constructive procedure allows finding coefficients of the regulator (25) for the case $w_{12} \neq 0$.
In Fig. 5, we present confidence ellipses for the Henon model (24) with $\mu=1.6, \varepsilon=0.001, s_{11}=$ $s_{22}=1$, fiducial probability $p=0.95$ and control inputs synthesizing the different stochastic sensitivity matrices

$$
\begin{gathered}
W_{1}=\left[\begin{array}{cc}
2 & w_{12} \\
w_{12} & 3
\end{array}\right], \quad W_{2}=\left[\begin{array}{cc}
4 & w_{12} \\
w_{12} & 5
\end{array}\right], \\
W_{3}=\left[\begin{array}{cc}
9 & w_{12} \\
w_{12} & 10
\end{array}\right]
\end{gathered}
$$

for two values, $w_{12}=0$ (Fig. 5(a)) and $w_{12}=1$ (Fig. 5(b)). As one can see, the attainability condition (26) restricts a variety of synthesized confidence ellipses essentially. However, even in the framework of this restriction, our regulator (25) allows localizing random states near the equilibrium $(\bar{x}, \bar{y})$.

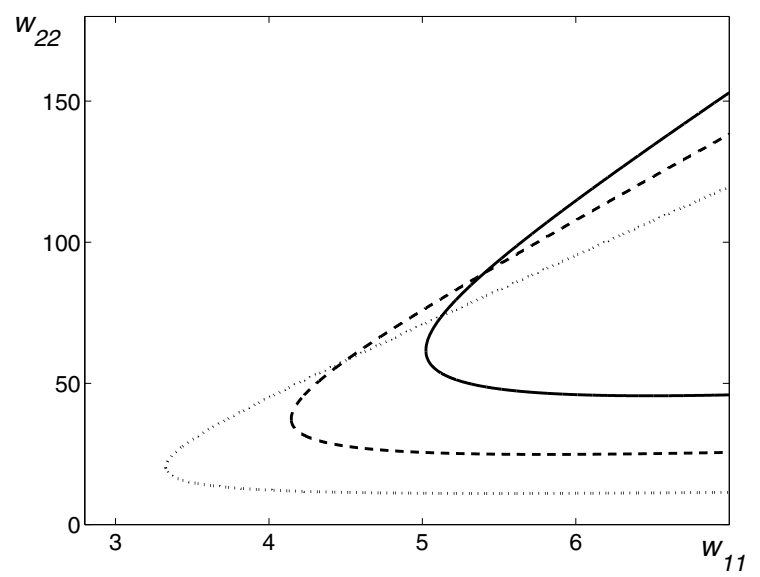

Fig. 6. Boundaries of attainability domains for the stochastic Henon model (28) with $s_{11}=s_{22}=1$ for $\mu=1.6$ (dotted), $\mu=2$ (dashed), $\mu=2.4$ (solid). 
4.3. Control III. Consider now the stochastically forced Henon system

$$
\begin{aligned}
& x_{t+1}=1-\mu x_{t}^{2}-0.5 y_{t}+\varepsilon \sigma_{1} \xi_{t}, \\
& y_{t+1}=x_{t}+u_{2, t}+\varepsilon \sigma_{2} \nu_{t}
\end{aligned}
$$

with the single control input $u_{2, t}$.

In this case,

$B=\left[\begin{array}{l}0 \\ 1\end{array}\right], \quad \operatorname{rank}[B, A B]=\operatorname{rank}\left[\begin{array}{cc}0 & -0.5 \\ 1 & 0\end{array}\right]=2$

so the pair $(A, B)$ is controllable.

We use a feedback regulator in the following form:

$$
u_{2}=k_{21}(x-\bar{x})+k_{22}(y-\bar{y}) .
$$

For the system 28, 29,

$$
\begin{gathered}
B^{+}=\left[\begin{array}{ll}
0 & 1
\end{array}\right], \quad B B^{+}=\left[\begin{array}{ll}
0 & 0 \\
0 & 1
\end{array}\right], \\
P=I-B B^{+}=\left[\begin{array}{ll}
1 & 0 \\
0 & 0
\end{array}\right] .
\end{gathered}
$$

From the attainability condition (12) it follows that for this case the elements of the assigned attainable stochastic sensitivity matrix $W$ must satisfy the system

$$
\begin{gathered}
w_{11} \geq s_{11}, \quad w_{22} \geq s_{22}, \\
\left(w_{11}-s_{11}\right)\left(w_{22}-s_{22}\right) \geq w_{12}^{2}, \\
\left(a^{2}(\mu)-1\right) w_{11}-a(\mu) w_{12}+0.25 w_{22}+s_{22}=0,
\end{gathered}
$$

where $a(\mu)=-2 \mu \bar{x}(\mu)=(3-\sqrt{9+16 \mu}) / 2$. Thus, the element $w_{12}$ is uniquely defined by the elements $w_{11}, w_{22}$ :

$$
w_{12}=\frac{a^{2}(\mu)-1}{a(\mu)} w_{11}+\frac{1}{4 a(\mu)} w_{22}+\frac{s_{22}}{a(\mu)} .
$$

Eliminating $w_{12}$ from (30), we get the following quadratic inequality for the attainability domain:

$$
\begin{aligned}
& w_{22}^{2}+8\left[\left(2 a^{2}+1\right) s_{11}-\left(a^{2}+1\right) w_{11}\right] w_{22} \\
+ & 16\left[\left(s_{11}+\left(a^{2}-1\right) w_{11}\right)^{2}+a^{2} s_{22}\left(w_{11}-s_{11}\right)\right] \leq 0 .
\end{aligned}
$$

Here, in contrast to the previous cases (Controls I and II), the attainability domain for the values $w_{11}, w_{22}$ depends essentially on the parameter $\mu$. This dependence is demonstrated in Fig. 6, where the boundaries of attainability domains are plotted for different values $\mu=$ 1.6 (dotted), $\mu=2$ (dashed), $\mu=2.4$ (solid). The corresponding attainability domains are arranged on the right of the boundaries.

Here, we focus on the problem of suppression of the stochastic and noise-induced chaotic oscillations via stochastic sensitivity synthesis. An uncontrolled Henon system with $\varepsilon=0.005, s_{11}=s_{22}=1$ demonstrates these regimes for $\mu=2$ (see Fig. 7(a), grey) and $\mu=2.4$ (see Fig. 7(b), grey).

To suppress stochastic oscillations of high amplitude for the system 21) with $\mu=2$, we use the regulator (29) which provides stability of the equilibrium $(\bar{x}, \bar{y})$ with small stochastic sensitivity. Set $w_{11}=4.5, w_{22}=$ 40. These values belong to the attainability domain (see Fig. 6). From (30) it follows that $w_{12}=-11.48$. Using (13) and (14) for synthesis of this stochastic sensitivity matrix, we construct the regulator 29) with feedback coefficients $k_{21}=4.622$ and $k_{22}=1.467$. This regulator provides oscillations of small amplitude near the stable equilibrium $(\bar{x}, \bar{y})$ (see Figs. 7(a) and 8(a)).

To suppress chaotic oscillations for the system (21) with $\mu=2.4$, we set $w_{11}=6, w_{22}=50$. These values belong to the corresponding attainability domain (see Fig. 6). From (30) it follows that $w_{12}=-15.52$. The same algorithm based on (13, (14) gives us feedback coefficients $k_{21}=4.752, k_{22}=1.342$. In Figs. 7(b) and $8(\mathrm{~b})$, it is shown that the regulator 29) with these coefficients suppresses noise-induced chaos successfully.

\section{Conclusion}

The problem of stabilization and control of stochastic equilibria for a nonlinear discrete-time stochastic system was studied. Our method of regulator synthesis is based on the stochastic sensitivity function technique. To realize this method, we have to describe attainability sets for the prescribed stochastic sensitivity matrix.

In this paper, a detailed geometrical description of attainability domains for various control inputs in general 2D nonlinear discrete-time stochastic systems was carried out. By the example of a stochastically forced controlled Henon system we described these attainability domains constructively and showed how these domains can be used for the stabilization of stochastic equilibria and suppression of noise-induced chaos.

\section{Acknowledgment}

This work was partially supported by the Ministry of Education and Science of Russia (1.1099.2011, 14.A18.21.0364) and RFBR (13-08-00069).

\section{References}

Albert, A. (1972). Regression and the Moore-Penrose Pseudoinverse, Academic Press, New York, NY.

Alexandrov, D. and Malygin, A. (2011). Nonlinear dynamics of phase transitions during seawater freezing with false bottom formation, Oceanology 51(6): 940-948.

Åström, K. (1970). Introduction to the Stochastic Control Theory, Academic Press, New York, NY. 


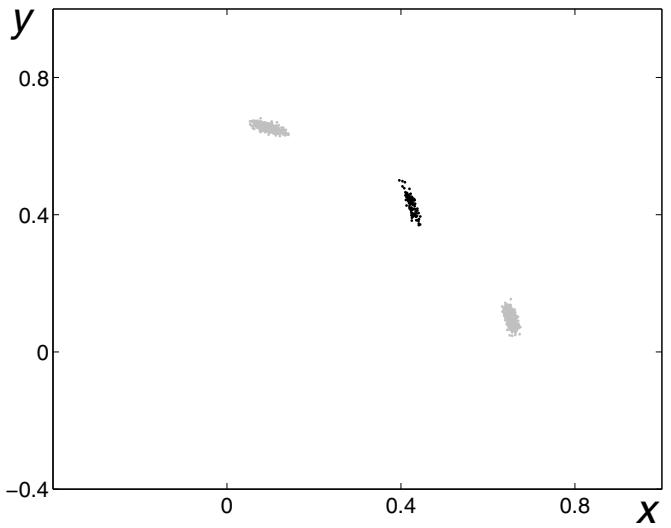

(a)

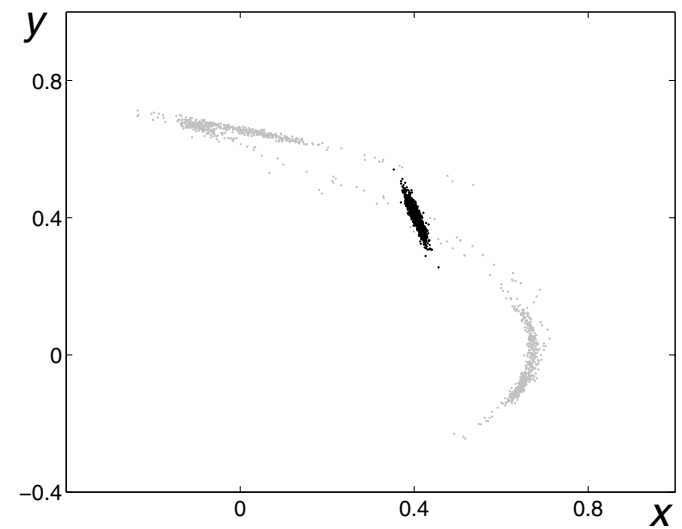

(b)

Fig. 7. Random states of the stochastic Henon model for $\varepsilon=0.005, s_{11}=s_{22}=1, p=0.95$ without control (grey) and with control (black) 28 synthesizing stochastic sensitivity matrices: $w_{11}=4.5, w_{22}=40, w_{12}=-11.48$ for $\mu=2$ (a), $w_{11}=6, w_{22}=50, w_{12}=15.52$ for $\mu=2.4$ (b).

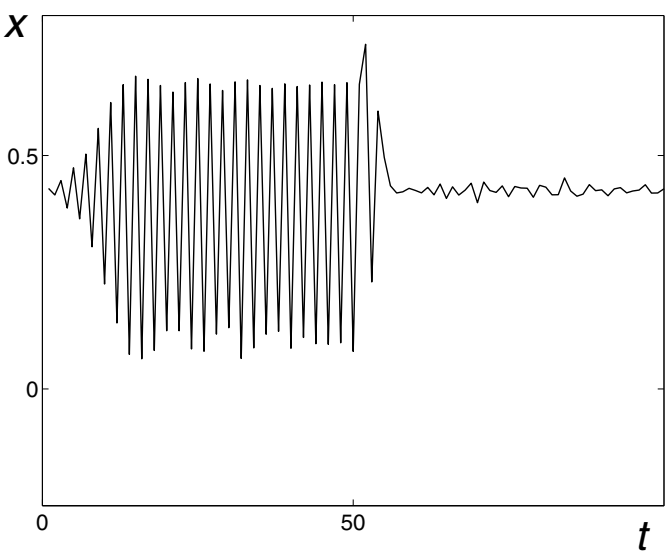

(a)

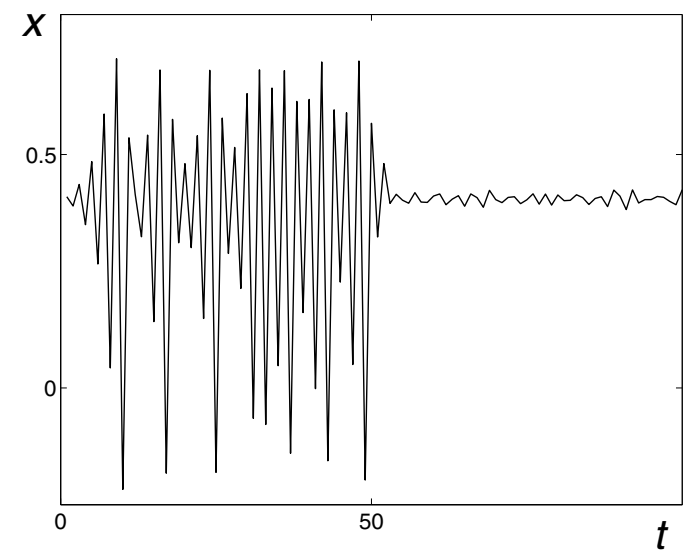

(b)

Fig. 8. Stabilization of stochastic oscillations for the Henon model for the set of parameters as in Fig. 7. Here the $x$-coordinate of the random trajectory is plotted and control is switched on at $t=50$.

Bashkirtseva, I. and Ryashko, L. (2000). Sensitivity analysis of the stochastically and periodically forced Brusselator, Physica A 278(1-2): 126-139.

Bashkirtseva, I. and Ryashko, L. (2005). Sensitivity and chaos control for the forced nonlinear oscillations, Chaos Solitons and Fractals 26(5): 1437-1451.

Bashkirtseva, I. and Ryashko, L. (2009). Constructive analysis of noise-induced transitions for coexisting periodic attractors of Lorenz model, Physical Review E 79(4): 041106-041114.

Bashkirtseva, I., Ryashko, L. and Stikhin, P. (2010). Noise-induced backward bifurcations of stochastic 3D-cycles, Fluctuation and Noise Letters 9(1): 89-106.

Bashkirtseva, I., Ryashko, L. and Tsvetkov, I. (2009). Sensitivity analysis of stochastic equilibria and cycles for the discrete dynamic systems, Dynamics of Continuous, Discrete and Impulsive Systems, Series A: Mathematical Analysis 17(4): 501-515.
Chen, G. and Yu, X.E. (2003). Chaos Control: Theory and Applications, Springer-Verlag, New York, NY.

Digailova, I. and Kurzhanskii, A. (2004). Attainability problems under stochastic perturbations, Differential Equations 40(11): 1573-1578.

Elaydi, S. (1999). An Introduction to Difference Equations, Springer-Verlag, New York, NY.

Fedotov, S., Bashkirtseva, I. and Ryashko, L. (2004). Stochastic analysis of subcritical amplification of magnetic energy in a turbulent dynamo, Physica A 342(3-4): 491-506.

Fradkov, A. and Pogromsky, A. (1998). Introduction to Control of Oscillations and Chaos, World Scientific Series of Nonlinear Science, Singapore.

Gao, J., Hwang, S. and Liu, J. (1999). When can noise induce chaos?, Physical Review Letters 82(6): 1132-1135.

Gassmann, F. (1997). Noise-induced chaos-order transitions, Physical Review E 55(3): 2215-2221. 
Goswami, B. and Basu, S. (2002). Transforming complex multistability to controlled monostability, Physical Review E 66(2): 026214-026223.

Henon, M. (1976). A two-dimensional mapping with a strange attractor, Communications in Mathematical Physics 50(1): 69-77.

Karthikeyan, S. and Balachandran, K. (2011). Constrained controllability of nonlinear stochastic impulsive systems, International Journal of Applied Mathematics and Computer Science 21(2): 307-316, DOI: 10.2478/v10006-011-0023-0.

Kučera, V. (1973). Algebraic theory of discrete optimal control for single-variable systems, III: Closed-loop control, $\mathrm{Ky}$ bernetika 9(4): 291-312.

Martínez-Zéregaa, B. and Pisarchik, A. (2002). Stochastic control of attractor preference in a multistable system, Communications in Nonlinear Science and Numerical Simulation 17(11): 4023-4028.

Matsumoto, K. and Tsuda, I. (1983). Noise-induced order, Journal of Statistical Physics 31(1): 87-106.

McDonnell, M., Stocks, N., Pearce, C. and Abbott, D. (2008). Stochastic Resonance: From Suprathreshold Stochastic Resonance to Stochastic Signal Quantization, Cambridge University Press, Cambridge.

Mil'shtein, G. and Ryashko, L. (1995). A first approximation of the quasipotential in problems of the stability of systems with random non-degenerate perturbations, Journal of Applied Mathematics and Mechanics 59(1): 47-56.

Nayfeh, A. and Balachandran, B. (2006). Applied Nonlinear Dynamics: Analytical, Computational, and Experimental Methods, Wiley, New York, NY.

Ott, E., Grebogi, C. and Yorke, J. (1990). Controlling chaos, Physical Review Letters 64(11): 1196-1199.

Ryagin, M. and Ryashko, L. (2004). The analysis of the stochastically forced periodic attractors for Chua's circuit, International Journal of Bifurcation and Chaos in Applied Sciences and Engineering 14(11): 3981-3987.
Ryashko, L. (1996). The stability of stochastically perturbed orbital motions, Journal of Applied Mathematics and Mechanics 60(4): 579-590.

Ryashko, L. and Bashkirtseva, I. (2011a). Analysis of excitability for the FitzHugh-Nagumo model via a stochastic sensitivity function technique, Physical Review E 83(6): 061109-061116.

Ryashko, L. and Bashkirtseva, I. (2011b). Control of equilibria for nonlinear stochastic discrete-time systems, IEEE Transactions on Automatic Control 56(9): 2162-2166.

Sanjuan, M. and Grebogi, C. (2010). Recent Progress in Controlling Chaos, World Scientific, Singapore.

Wonham, W. (1979). Linear Multivariable Control: A Geometric Approach, Springer-Verlag, Berlin.

Zhirabok, A. and Shumsky, A. (2012). An approach to the analysis of observability and controllability in nonlinear systems via linear methods, International Journal of Applied Mathematics and Computer Science 22(3): 507-522, DOI: 10.2478/v10006-012-0038-1.

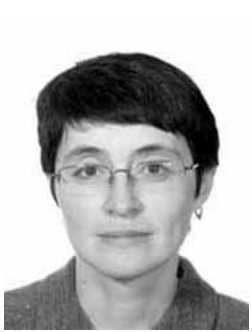

Irina Bashkirtseva is an associate professor at Ural Federal University. She received her Candidate of Science (Ph.D.) degree in differential equations from Ural State University (Ekaterinburg) in 1997. Her research interests include computational methods of nonlinear dynamic theory and stochastic control with applications to models exhibiting transitions from order to chaos.

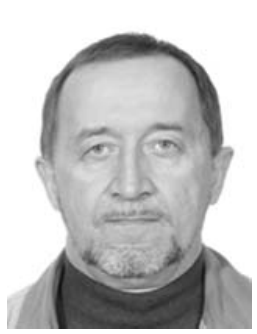

Lev Ryashko is a professor at Ural Federal University. He received his Candidate of Science (Ph.D.) degree in differential equations from Ural State University (Ekaterinburg) in 1980 and the Doctor of Science degree in system analysis and control from the Moscow Institute of Electronics and Mathematics in 2006. His research interests include noise-induced phenomena, stochastic bifurcations, and stabilization and control of nonlinear dynamic systems.

Received: 24 February 2012 Revised: 15 June 2012 